\title{
Aging of the Nervous, Locomotive, Cardiovascular, Respiratory, Digestive, Urinary and Hematopoirtic Systems
}

\author{
Heshmat SW Haroun* \\ Professor of Anatomy and Embryology, Egypt

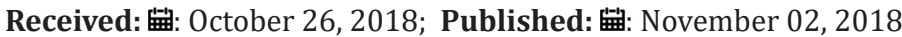 \\ *Corresponding author: Heshmat SW Haroun, Professor of Anatomy and Embryology, Egypt
}

\begin{abstract}
Aging is a slowly progressive natural process that is associated with adverse effects on different system organs. It induces changes in nearly every tissue in the body. Reactive oxygen species and mitochondrial damage are still the accepted two theories of aging. Age-related diseases include dementia and Alzheimer's disease, osteoarthritis and osteoporosis, hypertension, atherosclerosis and heart failure, chronic obstructive pulmonary disease and emphysema, hyperchlorhydria, hepatitis and hepatocellular carcinoma, glomerulosclerosis and chronic kidney disease, anemia and increased rate of carcinogenesis. Biomarkers of aging are available. Exercise, caloric restriction and drug dose modifications are recommended in the elderly.
\end{abstract}

Keywords: aging, nervous, locomotive, cardiovascular, respiratory, digestive, urinary, hematopoietic, drugs, cancer, biomarkers.

Abbreviations: AD: Alzheimer's Disease; ApoE: Apolipoprotein E; BBB: Blood-Brain Barrier; BCSFB: Blood-Cerebrospinal Fluid Barrier; CF: Cystic Fibrosis; CKD: Chronic Kidney Disease; COPD: Chronic Obstructive Pulmonary Disease; CR: Caloric Restriction; CVD: Cardiovascular Disease; EE: Energy Expenditure; GH: Growth Hormone; HF: Heart Failure; IGF-I: Insulin-like Growth Factor-1; JOA: Japanese Orthopaedic Association; NPC: Niemann-Pick Type C; RNS: Reactive Nitrogen Species; ROS: Reactive Oxygen Species; SA: Sino-Atrial, SSA: Senile Systemic Amyloidosis; TRH: Thyrotropin-Releasing Hormone

\section{Introduction}

Aging is described as a multifactorial, complex, progressive, natural and irreversible process that is injurious to the human body. Apart from immuno-senescence and age-related reproductive changes which we have previously reviewed [1,2], aging has a negative impact on nearly every tissue and organ in the body. Globally, increased longevity has been accompanied by increased incidence of age-related diseases which represent a costly burden on healthcare services. The etiology of aging is still not fully explained. Reactive oxygen species (ROS) and mitochondrial theories are the two valid theories of aging. Mitochondria are the major site for production of ROS in the cell. The vicious circle of oxidative stress theory includes that inside the mitochondria ROS induces mitochondrial damage and accumulation of oxidative damage particles which in turn increases the liberation of ROS, leading to more mitochondrial damage.

ROS impairs organ functions and increases the morbidity and mortality rates. ROS-related aging is mentioned to be ameliorated by general hygiene as well as environmental, nutritional and drug remedies [3-5]. Oxidative stress occurs when there is an oxidants/ antioxidants imbalance [6]. ROS and reactive nitrogen species
(RNS) are reported to be predominantly harmful but to lesser extent beneficial to tissues and organs [7]. Genetic and environmental factors contribute to the type and rate of functional decline with aging and to the development of age-associated diseases [8]. Air and water pollution, tobacco and alcohol, heat and cold, medications and chemicals, pesticides and radiation are mentioned as harmful factors in the elderly [9]. These toxicants induce oxidative stress leading to reduction of the regenerative capacity of the stem cells and enhancement of aging [10]. Autophagy is fundamental to maintain organ function through elimination of accumulated damaged particles; thus, prolonging lifespan [11].

Autophagy becomes less efficient with age [12]. Apolipoprotein E (ApoE) is greatly involved in age-related inflammation and atherosclerosis, and in rheumatoid arthritis, macular degeneration and cancer [13]. Oral or parental thyrotropin-releasing hormone (TRH) has proved to possess an antiaging property in old mice, it increased the testicular weight, structure and function and cured renal amyloid and hyaline degenerations [14]. Aging is reported to start at the age of 30-40. It induces alterations in organs of the nervous, locomotive, cardiovascular, respiratory, digestive, 
urinary, and hematopoietic systems. These organ changes could be aggravated into diseases that develop even in healthy elderly [15].

\section{Aging of the Nervous System}

Aging of the brain is assumed to be the referee of death, it finally leads to neurodegeneration and dementia [16]. Health condition of patients with highly impaired cognitive function and coming from unhealthy families is reported to be worse than that of individuals with less impaired cognition [17]. Aging worsens human brain function due to the release of ROS. Flavonoids in foods are antioxidants that delay the age-related decline of cognitive functions and the development of neurodegenerative diseases [18]. Blood-brain barrier (BBB) and blood-cerebrospinal fluid barrier (BCSFB) are documented to play a great role in the progression of age-associated neurodegeneration and Alzheimer's disease [19]. Age-related Alzheimer's disease (AD) is associated with silent microbleeds from small cerebral blood vessels and with stiffness of the aorta and great arteries [20]. The co-incidence of AD and osteoporosis increases in the elderly. In these two degenerative diseases. amyloid plaques and aggregations are deposited in the brain and in the skeletal elements. Vitamin D deficiency is diagnosed in $\mathrm{AD}$ or $\mathrm{AD}$ with osteoporosis [21].

Aging also results in disturbances in the autonomic nervous system. In aging rats, significant changes were demonstrated in the sympathetic noradrenergic innervation of the thymus, spleen, and mesenteric lymph nodes resulting in immunosuppression [22]. Age-related tachycardia, constipation, erectile dysfunction, fluid retention, systemic inflammation, type- 2 diabetes mellitus, insomnia, $\mathrm{AD}$, atherosclerosis, and cancer are consequences of loss of the parasympathetic function and unmasking of the basic sympathetic function [23]. Motor neurons and innervated muscle fibers exhibit age-related decline in number in addition to muscle fiber atrophy [24].

\section{Aging of the Locomotive System}

With aging, there is a diminished function of the skeletal muscles leading to decreased physical fitness, sarcopenia, frailty and eventually disability thus, independence and quality of life are affected in the elderly [25]. Sarcopenia is reported to begin at the fourth decade of life and to be featured by decreased muscle mass and strength, decreased total body protein and decreased muscle and mitochondrial protein synthesis. Mitochondria plays a crucial role in aging of the skeletal muscles [26]. Exercise is recommended in both young and older individuals, it increases muscle strength but does not correct all age-induced changes [27]. Vitamin D supplements are prescribed to the elderly to protect against the incidence of osteoporosis [28]. The Japanese Orthopaedic Association (JOA) provides loco-check and loco-training programs to minimize the incidence of the geriatric locomotive syndrome in the elderly suffering from joint disorders [29]. Aging is also associated with a decline in the levels of growth hormone (GH) and serum insulin-like growth factor-1 (IGF-I) resulting in osteoporosis and muscle atrophy. GH and IGF-I supplements are also recommended for the elderly [30].

\section{Aging of the Cardiovascular System}

Aging is associated with diminished elasticity of the arterial tree, decreased left ventricular diastole, declined function of the sino-atrial (SA) node and decreased effect of beta-adrenergic stimulation. Old patients with cardiovascular disease (CVD) are prone to develop heart failure (HF) [31]. HF is a multiple organ disease, it increases with aging, most likely due to increased insulin/ IGF-1 signalling and due to reduced functions of extracardiac organs [32]. Accumulation of ROS, with aging, increases the vulnerability to CVD and heart failure [33].

\section{Aging of the Respiratory System}

With aging, the respiratory system exhibits a decrease in gas exchange area, respiratory volumes and maximal oxygen consumption. Lung is one of the most important sites to show amyloid deposition in senile systemic amyloidosis (SSA). In pulmonary amyloidosis of the elderly, autoptic lung specimens demonstrated abnormal amyloid deposits around the bronchi, bronchioles, alveoli and pulmonary vessels but not around lymphatics [34]. In Niemann-Pick Type C (NPC) disease, caused by deficiency of either of NPC1 or NPC2 proteins, accumulation of unesterified cholesterol was observed in the lungs of mice; the disease had started early in the lungs, preceded other tissues and worsened with the advance of age [35].

Chronic obstructive pulmonary disease (COPD) is 2-3 times more frequent above the age of 60 years than in younger population. Aging elevates basal levels of inflammation and oxidative stress (inflammaging) and increases immuno-senescence [36]. Environmental gases, like cigarette smoke and kitchen vapours, are thought to enhance aging of the lungs via reduction of the resolution of inflammation, diminution of the anti-oxidant capacity and failure of disposal of damaged proteins; eventually leading to aggravation of COPD [37,38]. With aging, senile emphysema entails dilatation of the alveolar spaces and decreased lung elasticity, whereas COPD includes destruction of the alveolar walls and fibrosis of the respiratory passages [39]. Moreover, cystic fibrosis (CF) of lungs in adults is augmented with the advance of age leading to more decline in lung functions [40].

\section{Aging of the Digestive System}

Aging of the gastrointestinal tract in man is featured by prevalence of gastric atrophy and hypochlorhydria because of Helicobacter pylori infection and constipation. Constipation is thought to be mainly caused by the sedentary lifestyle and diet rather than by aging process. With aging there are decreased motility, weakened mucosal barrier and increased incidence of colonic cancer [41]. Aged patients with liver diseases exhibit significant decrease in liver volume and blood flow thus, increasing vulnerability to drug-induced hepatitis, viral hepatitis, autoimmune liver diseases and hepatocellular carcinoma [42]. Aging impairs the well-known regenerative capacity of the liver following partial hepatectomy due to decreased cell cycle as well as increased apoptosis and autophagy. Old mice, when compared to young ones, 
showed significant reduction in the relative liver/body weight ratio and marked elevation of liver cell damage markers at $48 \mathrm{~h}$ and $72 \mathrm{~h}$ after partial hepatectomy [43]. In rats, aging of the liver was observed to be associated with increased uptake function and decreased metabolic function of the hepatocytes but the excretory function was unaffected [44].

\section{Aging of the Kidneys}

Aged kidneys suffer from decreased renal blood flow and glomerular filtration rate, decreased tubular ability to conserve and dilute urine, and diminished ability to regulate the $\mathrm{pH}$ and serum sodium level. The age- related changes that occur in the kidneys are the results of natural organ senescence and atherosclerosis or diabetes mellitus that are commonly associated with aging [45]. Glomerulosclerosis as a part of vascular aging is the cause of frequent incidence of chronic kidney disease (CKD) in the elderly [46].

\section{Aging of the Hematopoietic System}

Aging induces alterations in the hematopoietic stem cells and progenitors leading to anemia, immuno-senescence, and myeloid diseases [47]. Organ-specific changes in the levels of ferritin (protein) and ferritin-bound iron have been reported pointing out to different pathways selected by different organs to keep pace with aging. In old rat spleens and livers, these levels were greater than in young rats whereas in the tongues, sternohyoid muscles and esophagus were lower than in young rats; in the larynx the levels were the same in both young and old animals [48].

\section{Aging and Caloric Restriction}

Total energy expenditure (EE), in man, is decreased with the advance of age due to reductions in activity and resting metabolic rate (RMR). The reducted RMR is most likely caused by a diminution in the mass of individual organs and reduction of the metabolic rates of individual tissues, favoring increased fat mass and reduced lean mass $[49,50]$. Advanced glycation end products (AGEs), especially produced in abnormal glucose metabolism and over-ingested in foods, are elevated in the circulation of the elderly leading to increased incidence of chronic diseases. Dietary restriction of AGEs and drug treatment with AGE-inhibitors and AGE-breakers are recommended [51]. Caloric restriction (CR) was found to prevent age-related diseases and to extend life-span in most animals, it has an anti-inflammatory effect, modulates mitochondrial activity and reduces oxidative damage [52]. Prolonged CR or rapamycin treatment had increased the proliferation of the hippocampal neural stem and progenitor cells (neurogenesis) in the brain dentate gyrus of aging female mice, leading to improvement of agerelated cognitive deficits [53]. Obesity enhances the development of age-related cardiovascular, respiratory and musculoskeletal diseases, type 2 diabetes mellitus, and some types of cancer [54]. Preadipocytes constitute about $15-50 \%$ of cells in fat tissue and can give rise to newly formed fat cells. Age-related changes in preadipocyte function can contribute to age-related fat tissue redistribution and metabolic diseases.[55].

\section{Aging and Cancer}

It has been observed that carcinogenesis is enhanced with age in both man and experimental animals. Aging facilitates the progression of cancer through: accumulation of damaged cells in the tissues and alterations in immune and endocrine homeostatic mechanisms [56,57].

\section{Aging and Drugs}

Elderly patients are greatly affected by the frequently prescribed psychotropic drugs (like benzodiazepines, antidepressants, antipsychotics and lithium), and cardiovascular drugs (like warfarin, diuretics and $\beta$-blockers). Dose adjustment of these pharmaceuticals is mandatory in the elderly [58]. Aging increases body fat and decreases total body water and lean mass. Consequently, hydrophilic drugs have a smaller while lipophilic drugs have a larger volume of distribution and a prolonged half-life. Added to this, aging is associated with a decline in renal function. Therefore, the old drug dosing aphorism " start low, go slow in elderly" becomes now valid [59].

\section{Aging Biomarkers}

Aging is typically associated with an increase in the production of pro-inflammatory cytokines and inflammatory markers (inflammaging) [60]. Biomarkers of aging are available to determine the biological age of individuals and organs, to assess the influence of various determinants and interventions on the rate and type of aging, and to evaluate life expectancy and future rate of morbidity [61]. There are biomarkers of the cardiovascular system, metabolic processes, inflammation, hypothalamic-pituitary activity, sympathetic nervous system, and individual organ functioning (like kidney, lung, liver and heart) [62].

\section{Conclusion}

An adequate knowledge of the etiology and adverse effects of aging is necessary to understand the derangement that occurs in individual system organs, to avoid unnecessary morbidity and disabilities, and to improve the quality life in the elderly.

\section{References}

1. Heshmat SWH (2018) Aging of reproductive systems- A review article. IGWHC 1(4): ID:000117.

2. Haroun HSW (2018) Aging of thymus gland and immune system- Mini review. MOJ Anat \& Physiol, 5(3): 178-181.

3. Romano AD, Serviddio G, de Matthaeis A, Bellanti F, Vendemiale G (2010) Oxidative stress and aging. J Nephrol 23 Suppl 15: S29-36.

4. Le Gall JY, Ardaillou R (2009) The biology of aging. Bull Acad Natl Med 193(2): 365-402; discussion 402-404.

5. Singh KK (2006) Mitochondria damage checkpoint, aging, and cancer. Ann N Y Acad Sci 1067: 182-190.

6. Desai KM, Chang T, Wang H, Banigesh A, Dhar A, et al. (2010) Oxidative stress and aging: is methylglyoxal the hidden enemy? Can J Physiol Pharmacol 88(3): 273-284.

7. Oliveira BF, Nogueira-Machado JA, Chaves MM (2010) The role of oxidative stress in the aging process. Scientific World Journal 10: 11211128. 
8. Walker LC, Herndon JG (2010) Mosaic aging. Med Hypotheses 74(6): 1048-1051.

9. Jafri AB (2011) Aging and toxins. Clin Geriatr Med 27(4): 609-628.

10. Hodjat M, Rezvanfar MA, Abdollahi M (2015) A systematic review on the role of environmental toxicants in stem cells aging. Food Chem Toxicol 86: 298-308.

11. Yamaguchi O, Otsu K (2012) Role of autophagy in aging. J Cardiovasc Pharmacol 60(3): 242-247.

12. Rezzani R, Stacchiotti A, Rodella LF (2012) Morphological and biochemical studies on aging and autophagy. Ageing Res Rev 11(1): 1031.

13. Bonomini F, Filippini F, Hayek T, Aviram M, Keidar S, et al. (2010) Apolipoprotein E and its role in aging and survival. Exp Gerontol 45(2): 149-157.

14. Pierpaoli W (2013) Aging-reversing properties of thyrotropin-releasing hormone. Curr Aging Sci 6(1): 92-98.

15. Wieczorowska Tobis K (2008) Organ alterations due to aging. Pol Arch Med Wewn 118: 63-69.

16. Wyss-Coray T (2016) Ageing, neurodegeneration and brain rejuvenation. Nature 539(7628): 180-186.

17. Blazeković-Milaković S, Kern J, Kulenović M (2000) Health status as geneologic burden in aging process. Coll Antropol 24(1): 79-89.

18. Schmitt-Schillig S, Schaffer S, Weber CC, Eckert GP, Müller WE (2005) Flavonoids and the aging brain. J Physiol Pharmacol 56 Suppl 1: 23-36.

19. Gorlé N, Van Cauwenberghe C, Libert C, Vandenbroucke RE (2016) The effect of aging on brain barriers and the consequences for Alzheimer's disease development. Mamm Genome 27(7-8): 407-420.

20. Stone J, Johnstone DM, Mitrofanis J, O’Rourke M (2015). The mechanical cause of age-related dementia (Alzheimer's disease): the brain is destroyed by the pulse. J Alzheimers Dis 44(2): 355-373.

21. Chen YH, Lo RY (2017) Alzheimer's disease and osteoporosis. Ci Ji Yi Xue Za Zhi 29(3): 138-142.

22. Thyaga Rajan S, Madden KS, Teruya B, Stevens SY, Felten DL, et al. (2011) Age-associated alterations in sympathetic noradrenergic innervation of primary and secondary lymphoid organs in female Fischer 344 rats. J Neuroimmunol 233(1-2): 54-64.

23. Lee PY, Yun AJ, Bazar KA (2004) Conditions of aging as manifestations of sympathetic bias unmasked by loss of parasympathetic function. Med Hypotheses 62(6): 868-870.

24. Deschenes MR (2011) Motor unit and neuromuscular junction remodelling with aging. Curr Aging Sci 4(3): 209-220.

25. Garatachea N, Lucia A (2013) Genes, physical fitness and ageing. Ageing Res Rev 12(1): 90-102.

26. Figueiredo PA, Mota MP, Appell HJ, Duarte JA (2008) The role of mitochondria in aging of skeletal muscle. Biogerontology 9(2): 67-84.

27. Karakelides H, Nair KS (2005) Sarcopenia of aging and its metabolic impact. Curr Top Dev Biol 68: 123-148.

28. Lanske B, Razzaque MS (2007) Vitamin D and aging: old concepts and new insights. J Nutr Biochem 18(12): 771-777.

29. Nakashima Y, Okazak K, Nakayama K, Okada S, Mizu uchi H (2017) Bone and Joint Diseases in Present and Future. Fukuoka Igaku Zasshi 108(1): $1-7$.

30. Hoffman AR, Lieberman SA, Ceda GP (1992) Growth hormone therapy in the elderly: implications for the aging brain. Psychoneuroendocrinology 17(4): 327-333.

31. Shinmura K (2016) Cardiac Senescence, Heart Failure, and Frailty: A Triangle in Elderly People. Keio J Med 65(2): 25-32.
32. Shioi T, Inuzuka Y (2012) Aging as a substrate of heart failure. J Cardiol 60(6): 423-428

33. Narasimhan M, Rajasekaran NS (2017) Cardiac Aging - Benefits of Exercise, Nrf2 Activation and Antioxidant Signaling. Adv Exp Med Biol 999: 231-255.

34. Ueda M, Ando Y, Haraoka K, Katsuragi S, Terasaki Y, et al. (2006) Aging and transthyretin-related amyloidosis: pathologic examinations in pulmonary amyloidosis. Amyloid 13(1): 24-30.

35. Ramirez CM, Lopez AM, Le LQ, Posey KS, Weinberg AG, et al. (2014) Ontogenic changes in lung cholesterol metabolism, lipid content, and histology in mice with Niemann-Pick type C disease. Biochim Biophys Acta 1841(1): 54-61.

36. Mac Nee W (2016) Is Chronic Obstructive Pulmonary Disease an Accelerated Aging Disease? Ann Am Thorac Soc 13(5): S429-S437.

37. Ito K Mercado N (2014) STOP accelerating lung aging for the treatment of COPD. Exp Gerontol 59: 21-27.

38. Mac Nee W (2009) Accelerated lung aging: a novel pathogenic mechanism of chronic obstructive pulmonary disease (COPD). Biochem Soc Trans 37(Pt 4): 819-823.

39. Mercado N, Ito K, Barnes PJ (2015) Accelerated ageing of the lung in COPD: new concepts. Thorax 70(5): 482-489.

40. Simmonds NJ (2013) Ageing in cystic fibrosis and long-term survival. Paediatr Respir Rev 14(1): 6-9.

41. Englander EW (2005) Gene expression changes reveal patterns of aging in the rat digestive tract. Ageing Res Rev 4(4): 564-578.

42. Tajiri K, Shimizu Y (2013) Liver physiology and liver diseases in the elderly. World J Gastroenterol 19(46): 8459-8467.

43. Enkhbold C, Morine Y, Utsunomiya T, Imura S, Ikemoto T, et al. (2015) Dysfunction of liver regeneration in aged liver after partial hepatectomy. J Gastroenterol Hepatol 30(7): 1217-1224.

44. Jourdan M, Vaubourdolle M, Cynober L, Aussel C (2004) Effect of aging on liver functions-an experimental study in a perfused rat liver model. Exp Gerontol 39(9): 1341-1346.

45. Glassock RJ, Rule AD (2012) The implications of anatomical and functional changes of the aging kidney: with an emphasis on the glomeruli. Kidney Int 82(3): 270-277.

46. Abdelhafiz AH, Brown SH, Bello A, El Nahas M (2010) Chronic kidney disease in older people: physiology, pathology or both? Nephron Clin Pract 116(1): c19-24

47. Gazit R, Weissman IL, Rossi DJ (2008) Hematopoietic stem cells and the aging hematopoietic system. Semin Hematol 45(4): 218-224.

48. Bulvik BE, Berenshtein E, Konijn AM, Grinberg L, Vinokur V, et al. (2012) Aging is an organ-specific process: changes in homeostasis of iron and redox proteins in the rat. Age (Dordr) 34(3): 693-704.

49. St Onge MP, Gallagher D (2010) Body composition changes with aging: the cause or the result of alterations in metabolic rate and macronutrient oxidation? Nutrition 26(2): 152-155.

50. Manini TM (2010) Energy expenditure and aging. Ageing Res Rev 9(1): $1-11$.

51. Semba RD, Nicklett EJ, Ferrucci L (2010) Does accumulation of advanced glycation end products contribute to the aging phenotype? J Gerontol A Biol Sci Med Sci 65(9): 963-975.

52. López Lluch G, Navas P (2016) Calorie restriction as an intervention in ageing. J Physiol 594(8): 2043-2060.

53. Park JH, Glass Z, Sayed K, Michurina TV, Lazutkin A, et al. (2013) Calorie restriction alleviates the age-related decrease in neural progenitor cell division in the aging brain. Eur J Neurosci 37(12): 1987-1993. 
54. Tzanetakou IP, Katsilambros NL, Benetos A, Mikhailidis DP, Perrea DN (2012) Is obesity linked to aging? : adipose tissue and the role of telomeres. Ageing Res Rev 11(2): 220-229.

55. Sepe A, Tchkonia T, Thomou T, Zamboni M, Kirkland JL (2011) Aging and regional differences in fat cell progenitors - a mini-review. Gerontology 57(1): 66-75.

56. Anisimov VN (2003) The relationship between aging and carcinogenesis: a critical appraisal. Crit Rev Oncol Hematol 45(3): 277-304.

57. Anisimov VN, Sikora E, Pawelec G (2009) Relationships between cancer and aging: a multilevel approach. Biogerontology 10(4): 323-338.

58. Trifirò G, Spina E (2011) Age-related changes in pharmacodynamics: focus on drugs acting on central nervous and cardiovascular systems. Curr Drug Metab 12(7): 611-620.

ISSN: 2574-1241

DOI: $10.26717 / B J S T R .2018 .10 .001997$

Heshmat SW Haroun. Biomed J Sci \& Tech Res

(C) (P) This work is licensed under Creative Commons Attribution 4.0 License

Submission Link: https://biomedres.us/submit-manuscript.php
59. Shi S, Klotz U (2011) Age-related changes in pharmacokinetics. Curr Drug Metab 12(7): 601-610.

60. Cevenini E, Caruso C, Candore G, Capri M, Nuzzo D, et al. (2010) Agerelated inflammation: the contribution of different organs, tissues andsystems. How to face it for therapeutic approaches. Curr Pharm Des 16(6): 609-618.

61. Mooradian AD (1992) Biological and functional definition of the older patient: the role of biomarkers of aging. Oncology (Williston Park) 6(2 Suppl): 39-44.

62. Crimmins E, Vasunilashorn S, Kim JK, Alley D (2008) Biomarkers related to aging in human populations. Adv Clin Chem 46: 161-216.

$\begin{array}{ll}\text { BIOMEDICAL } & \text { Assets of Publishing with us } \\ \text { RESEARCHES } & \text { - Global archiving of articles } \\ \text { - Immediate, unrestricted online access }\end{array}$

